\title{
BRIGHT POINTS (MOUSTACHES) AND ARCH FILAMENTS IN YOUNG ACTIVE REGIONS
}

\author{
A. BRUZEK \\ (Fraunhofer Institut, Freiburg i. Br., Germany)
}

\begin{abstract}
A brief survey is given on the main characteristics of 'bright points' and of arch filaments in young bipolar spotgroups. Observations show that both features are closely associated, thus indicating that they have a common cause. It is suggested that the propagation of expanding and increasing activeregion magnetic fields through the chromosphere might be that cause.
\end{abstract}

\section{Introduction}

The present paper is concerned with two apparently quite different phenomena of solar activity which, however, as will be shown, are closely associated in young active regions. These two phenomena are the 'bright points' (which are also called 'moustaches' or 'bombs') and a particular type of small dark filaments which will be called 'arch filaments'. The results presented here were derived from observations carried out with a Halle $0.5 \AA \mathrm{H} \alpha$ filter at the domeless Coudé refractor at the Anacapri station of the Fraunhofer Institut in summer 1966. Photographs of about 20 active regions of different sizes and types were taken during their disk passage in the $\mathrm{H} \alpha$ center and at $H \alpha \pm 0.5$ and $H \alpha \pm 1.0 \AA$. Exposure times on Eastman Kodak 4E were $1 / 15-1 / 8 \mathrm{sec}$. The diameter of the Sun's image on the $35-\mathrm{mm}$ negative is $150 \mathrm{~mm}$.

\section{Bright Points}

Bright points have been investigated before by a number of authors (Beckers, 1964; Howard and Harvey, 1964; Koval, 1962, 1964, 1965, 1966; Lyot, 1941; McMath et al., 1960; Severny, 1957, 1959; Severny and Koval, 1961). Their main characteristic is a Balmer-line profile with a minimum at the center of the line, a maximum at about +1.0 and $-1.0 \AA$ distance from the line centre and wings $5-10 \AA$ wide. Due to that profile the bright points are invisible in the line centre and best visible at $\pm 1.0 \AA$. If the Halle filter is tuned to the $1.0 \AA$ position two $0.5 \AA$ bands centered on $\mathrm{H} \alpha+1.0$ and $\mathrm{H} \alpha-1.0 \AA$ respectively are transmitted concurrently. This most favorable position was used in general in photographing the bright points at Anacapri.

The results of the analysis of the Anacapri filtergrams partly agree, partly disagree with those of previous investigations. A summary only is given here. 
It was found that bright points occur in all active stages of active regions and are absent around old, strictly unipolar spots and in decaying plage regions. They are very conspicuous and numerous in large, complex spotgroups, where they are found at the outer edge of the penumbra of large spots, often at the ends of surgelike dark filaments, and also close to smaller spots (Figure 1). Bright points are a prominent feature also in the interspot region of many small and medium-sized bipolar spotgroups (Figure 2). They even occur in the very first stage of development of an active region when it still consists of a small bright $\mathrm{H} \alpha$ plage without a spot.

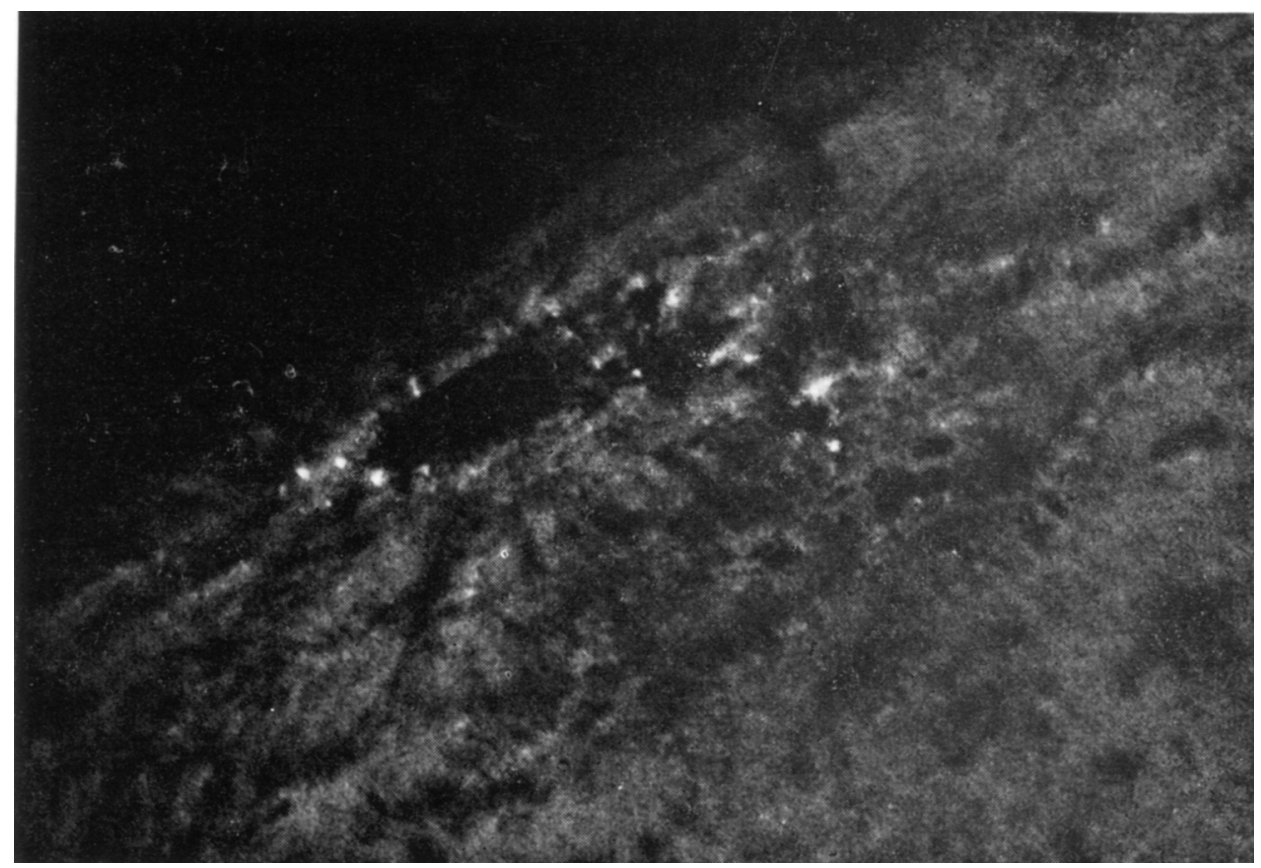

FIG. 1. Bright points in a large spotgroup near the West limb; $(H \alpha \pm 1$ A)-filtergram, August 29, 1966,1040 UT covers $11 \times 16\left(10^{4} \mathrm{~km}\right)^{2}$ of the sun.

The diameters of bright points as measured on the negative or on a hard print range from $1-5 \mathrm{sec}$ of arc (a typical value is 2 ."5). These values are determined apparently rather by the resolving power of the image-forming system (including the Earth's atmosphere) than by the true dimensions of the source of emission which probably are less than 1 ". During periods of excellent seeing a very large number of tiny bright points become visible in the eyepiece, as was also observed by Beckers (1964). Their number may exceed 100 by far in larger spotgroups and their size certainly is $\ll 1^{\prime \prime}$. In one large spotgroup up to 40 bright points of medium size and up to 10 of large apparent size and brightness may be present simultaneously. 
(a)
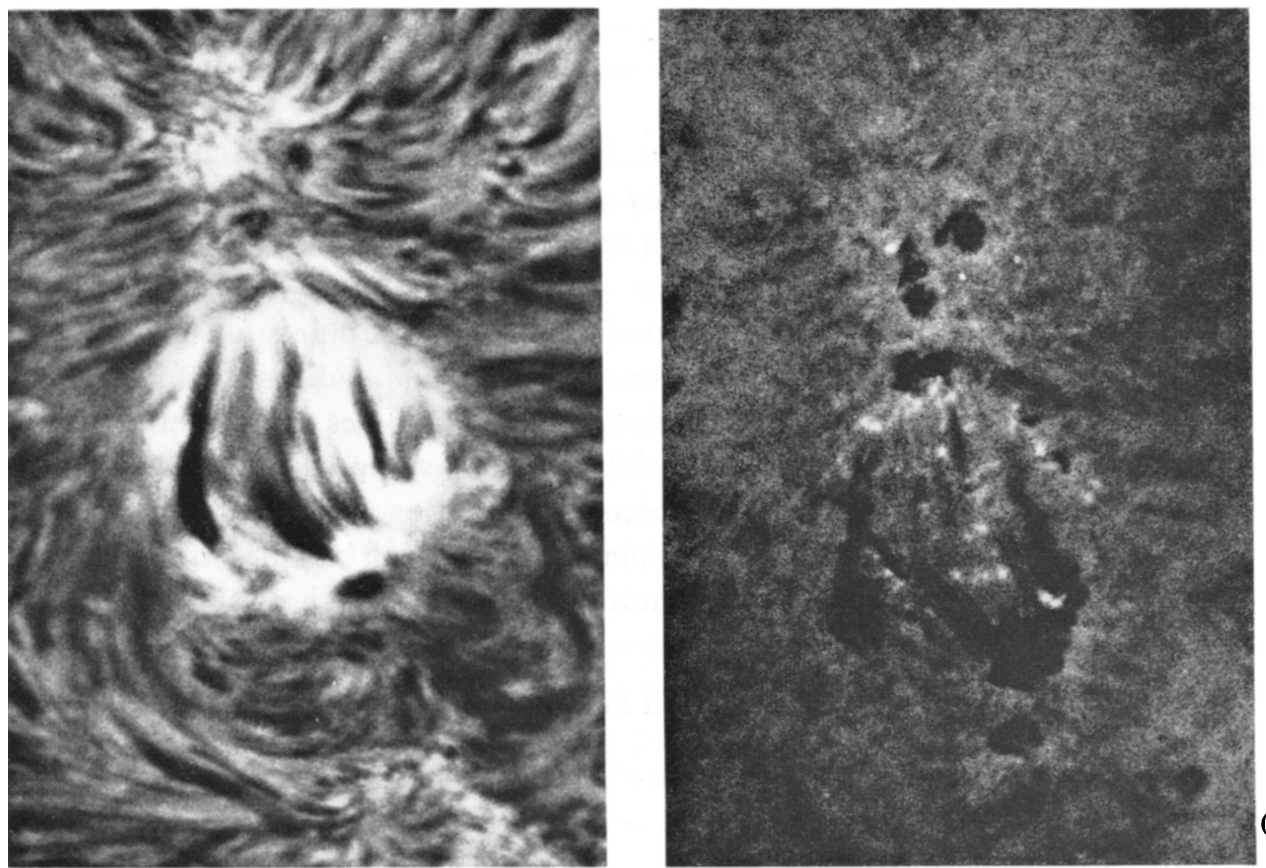

(b)
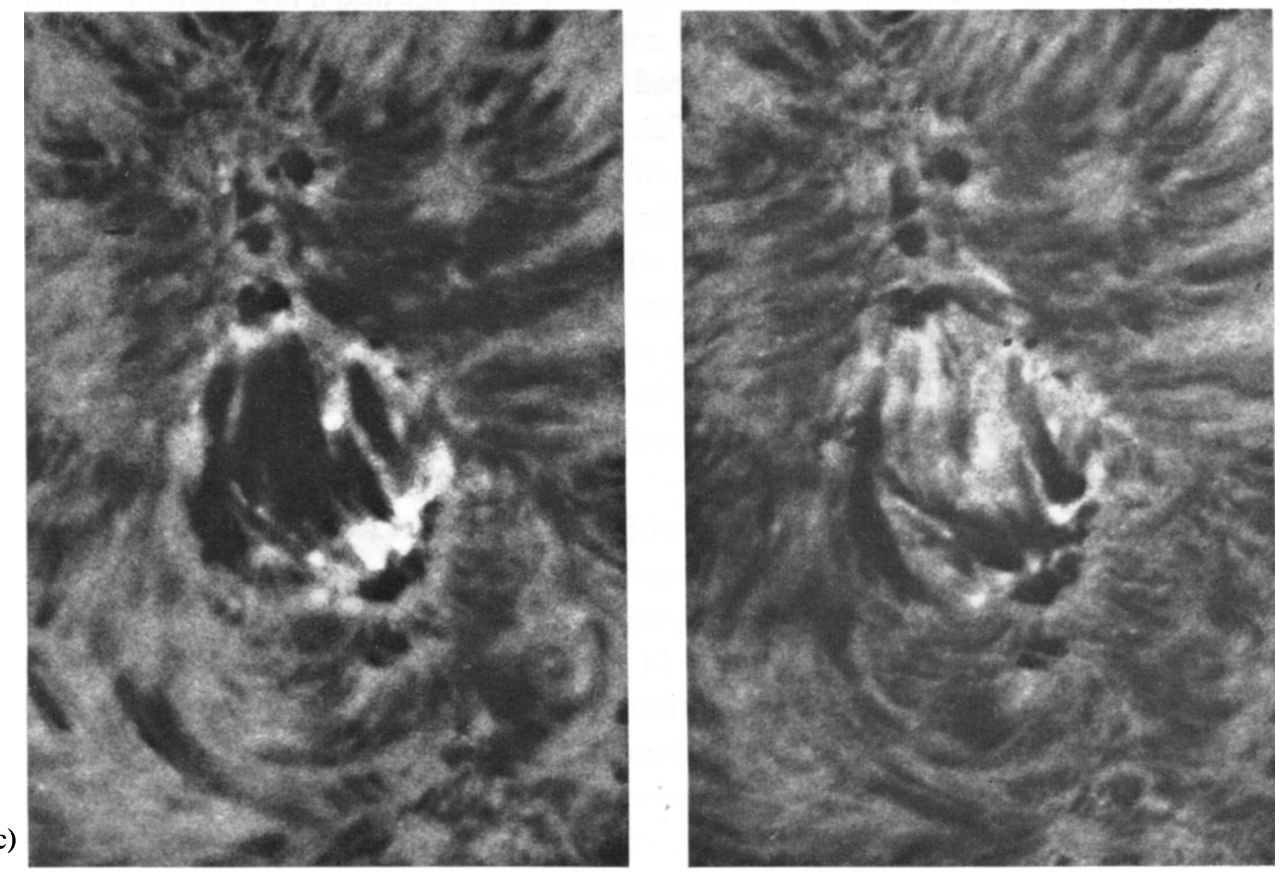

(d)

Fig. 2. Bright points and arch-filament system in a small bipolar spotgroup (type B) August 12, 1966, $1035 U T$; photographs taken (a) in $H \alpha$, , (b) at $H \alpha \pm 1 A$, (c) at $H \alpha-0.5 A$, (d) at $H \alpha+0.5 A$. The spots are visible in the 'wing filtergrams' $(b)-(d)$; bright points are seen best in the \pm 1 A filtergram (b). Mass flow inside the dark filaments directed from the top to the bottom of the frame and an expansion of the arches towards the observer are indicated by the differences between the -0.5 and $+0.5 \AA$ pictures (frames $(c)$ and $(d))$. The frames cover $75 \times 110\left(10^{3} \mathrm{~km}\right)^{2}$ of the Sun's disk. 
The brightness of well-defined bright points as measured on calibrated photographs was $115-150 \%$ of the brightness of the undisturbed surrounding in the same wavelength (typical $125 \%$ ).

The typical lifetime of bright points is $25 \mathrm{~min}$; extreme values found were 4 and $>120 \mathrm{~min}$. No strict recurrence tendency was found. New bright points frequently appeared quite close to but not exactly at the place of a vanished bright point; sometimes close pairs were formed.

My observations do not confirm the preference of bright points for the vicinity of the neutral line which Koval (1966) claims to have found. I did not find either any evidence for a direct relation between the occurrence of flares and bright points.

Furthermore, occurrence of bright points in young spotgroups does not indicate whether or not the active region will become a large one: many and conspicuous bright points were observed in fast developing regions, which became large spotgroups within a few days as well as in very short-lived regions which disappeared after a lifetime of a few days.

\section{Arch Filaments}

In young active regions a peculiar type of active, arch-shaped filaments is observed, which show opposite Doppler shifts at their opposite ends indicating a mass flow from and into the chromosphere along the filament. Single filaments of this type are present in the very youngest active regions containing a small plage and a few spots just born. They were studied by Waldmeier (1937) and Ellison (1944).

Groups or systems of many arch-shaped filaments occur in the interspot region of small and medium bipolar spotgroups of Zurich type B, C, and sometimes D (Bruzek, 1967). They connect the innermost spots of opposite magnetic polarity, thus crossing the neutral line and obviously following magnetic-field lines. Sometimes the ascending part of the individual filaments is found to be much larger than the descending one (Figure 2). That obviously means that there is not only a mass flow along the filament but also an expansion or material growth of the individual arches. The corresponding velocities are about 50 and $20 \mathrm{~km} / \mathrm{sec}$. Observations at and near the solar limb show that the arches are rather flat. Their vertices are $2000-7000 \mathrm{~km}$ high; they exceed $10000 \mathrm{~km}$ in exceptional cases only.

The lifetime of the individual filaments of a system may be estimated to 20-30 min. They undergo, however, noticeable changes within $10 \mathrm{~min}$, whereas the general configuration of the system as a whole remains almost unchanged for several hours. Arch-filament systems may be present in a spotgroup for several days. They are modified as soon as the spot configuration changes.

It must be pointed out that the arch-filament systems are quite different from loopprominence systems investigated by Dodson (1961) and Bruzek $(1962,1964)$. They differ in origin, development, inside motions and final height, they are similar in their connection with the magnetic field. 


\section{The Relation between Bright Points and Arch Filaments}

In Figure 2 a small bipolar spotgroup of type B is photographed in four wavelength bands. It shows that bright points occur almost only in the interspot region covered or bridged by the arch filaments. The bright points are situated frequently near the ends of the arches but are also visible between the filaments; it is likely that a number of bright points is even hidden below the arches. There is hardly a bright point found outside the spotgroup.

From a number of observations it became evident that bright points do not occur in young spotgroups unless there are arch filaments. In one case two bipolar groups of approximately the same age and size were present on the solar disk. In one of them both features, bright points as well as arch filaments existed, in the other one none of them was visible at the same time. In other cases a spotgroup contained neither arch filaments nor bright points on one day, but both of them on the following day.

The close connection between bright points and arch filaments is also true for the very first stages of an active region. A few bright points were found near a small arch filament or at its ends in two tiny active regions which had just appeared several hours ago. The active region consisted of no more than a very small bright plage (rapidly growing) and a short arch filament; no spot was yet visible.

\section{Conclusions}

The close relation between bright points and arch filaments suggests a common cause of both features. It is very likely that the arch filaments are related to the combined transport of matter and increasing magnetic field into higher levels during the growth of the spotgroup (Bruzek, 1967). We may therefore conclude that occurrence of bright points depends on either the propagation of the field through the low chromosphere or the ejection of matter. The latter suggestion is supported by the association of bright points with surgelike features in large spotgroups. The possible coincidence of bright points and surges with 'magnetic dots' as suggested by observations of Beckers and Schröter (1968) may be an important point for the understanding of the phenomena. A thorough discussion on the nature of bright points and their relation to the arch filaments has to be postponed, however, until more data are available.

\section{References}

Beckers, J. M. (1964) Thesis Utrecht, p. 33.

Beckers, J. M., Schröter, E. (1968) the present volume, p. 178.

Bruzek, A. (1962) Z. Astrophys., 54, 225.

Bruzek, A. (1964) Astrophys. J., 140, 746.

Bruzek, A. (1967) Solar Phys. 2, 451.

Dodson, H.W. (1961) Proc.nat. Acad. Sci. Am., 47, 901.

Ellison, M.A. (1944) Mon. Not. R. astr. Soc., 104, 22. 
Howard, R., Harvey, J. W. (1964) Astrophys. J., 139, 1328.

Koval, A. N. (1962) Izv. Krim. astrofiz. Obs., 28, 241.

Koval, A.N. (1964) Izv. Krim. astrofiz. Obs., 32, 32.

Koval, A.N. (1965) Izv. Krim. astrofiz. Obs., 33, 138.

Koval, A.N. (1966) Izv. Krim. astrofiz. Obs., 34, 278.

Lyot, B. (1941) Ann. Astrophys., 7, 48.

McMath, R.R., Mohler, O.C., Dodson, H.W. (1960) Proc. nat. Acad. Sci. Am., 46, 168.

Severny, A. B. (1957) Izv. Krim. astrofiz. Obs., 17, 129.

Severny, A.B. (1959) Izv. Krim. astrofiz. Obs., 21, 131.

Severny, A. B., Koval, A. N. (1961) Izv. Krim. astrofiz. Obs., 26, 3.

Waldmeier, M. (1937) Z. Astrophys., 14, 91.

\section{DISCUSSION}

J. Evans: You have shown that points and arches tend to occur in the same areas within new active centres. I want to be quite clear, however, about whether or not the locations of points are related to the specific geometry of individual arches. Do arches usually or always begin or terminate in a point? Or is there any other such relation?

Bruzek: Many of the arches begin and terminate, or either alone, in 'bright points', but far from all. On the other hand there are many 'bright points' between arches and at no foot of a filament at all.

Krüger: I should like to ask you if somewhat is known about the frequency of occurrence at bright points during the 11-year solar cycle; especially, is this phenomenon more characteristic for the beginning of a new solar cycle or not?

Bruzek: There is no known dependence of occurrence of 'bright points' on the 11-year cycle. 